LA-UR- $98-456 \%$

Title:

Author(s):

Submitted to:

VIIIth International Conference on Megagauss Field Generation and Related Topics, October 18-23, 1998; Tallahassee, F1orida, USA
A Review of U.S. High Explosive Pulsed

Power Systems

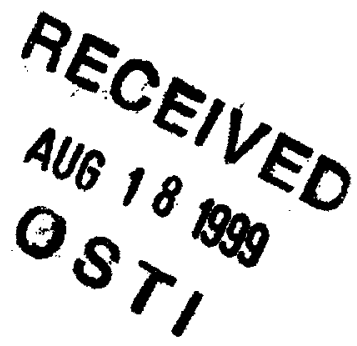

James H. Goforth

\section{Los Alamos}

NATIONAL LABORATORY

Los Alamos National Laboratory, an affirmative action/equal opportunity employer, is operated by the University of California for the U.S. Department of Energy under contract W-7405-ENG-36. By acceptance of this article, the publisher recognizes that the U.S. Government retains a nonexclusive, royalty-free license to publish or reproduce the published form of this contribution, or to allow others to do so, for U.S. Government purposes. The Los Alamos National Laboratory requests that the publisher identify this article as work performed under the auspices of the U.S. Department of Energy. 


\section{DISCLAIMER}

This report was prepared as an account of work sponsored by an agency of the United States Government. Neither the United States Government nor any agency thereof, nor any of their employees, make any warranty, express or implied, or assumes any legal liability or responsibility for the accuracy, completeness, or usefulness of any information, apparatus, product, or process disclosed, or represents that its use would not infringe privately owned rights. Reference herein to any specific commercial product, process, or service by trade name, trademark, manufacturer, or otherwise does not necessarily constitute or imply its endorsement, recommendation, or favoring by the United States Government or any agency thereof. The views and opinions of authors expressed herein do not necessarily state or reflect those of the United States Government or any agency thereof. 


\section{DISCLAIMER}

Portions of this document may be illegible in electronic image products. Images are produced from the best available original document. 


\title{
A Review of U. S. High Explosive Pulsed Power Systems
}

\author{
J. H. Goforth, University of California, Los Alamos National
}

Laboratory

Introduction

High explosive pulsed power (HEPP) is a specialized subset among pulsed power endeavors which takes advantage of the very high energy density available in both magnetic fields and high explosives (HE). To introduce basic concepts, I will divide HEPP components into generators (magnetic field (B) or current (I)) and switches. Magnetic field and current generators start with magnetic field trapped in a conducting volume. Magnetic flux can be expressed as either LI or BA, where $\mathrm{L}$ and $\mathrm{A}$ (inductance and cross sectional area) are both geometry dependent circuit properties. In a purely inductive circuit, flux is conserved, so $L_{1} I_{1}=L_{2} I_{2}$ or $B_{1} A_{1}=B_{2} A_{2}$. In our technique, $H E$ is used to propel circuit elements that perform work against the trapped magnetic field as $\mathrm{L}$ or $\mathrm{A}$ is reduced, yielding increased $\mathrm{I}$ or B. Throughout this paper, I will use the term flux compression generator (FCG) for these devices, although the reader will find a variety of acronyms in the literature. A good primer on FCG's is by Fowler et al. .

HE is also used to provide opening and closing switches for HEPP circuits. Closing switches do not require great sophistication, and we will not discuss them here. Opening switches typically use the energy of $\mathrm{HE}$ to rapidly reduce the current carrying cross section of a particular circuit element, and often require sophisticated detonation systems to match the contour of that element (e. g. cylindrical). This may either cause a direct increase in resistance or create the circumstance in which the remainder of the material fuses due to ohmic effects. Many good papers on explosive-driven opening switches can be found in previous Megagauss conference proceedings, and these are also a good source for information regarding HEPP endeavors outside the U. S., which is beyond the scope of this paper.

Max Fowler is discussing the history of HEPP at Los Alamos in this conference, and in this paper I will summarize current HEPP work in the U.S. From the perspective of the U.S. at large, HEPP activities began at Los Alamos in the mid50. The field became very active during the 60 's and early 70 's, with efforts at Los Alamos, Lawrence Livermore National Laboratory (LLNL), and Sandia National Laboratory (SNL), and considerable interest from Air Force Research Laboratory (ARFL) and other DoD laboratories. Interest in HEPP waned in the 70 's and $80^{\prime}$ 's for a variety of reasons, and LLNL and SNL effectively ceased HEPP activities by the end of the 80's. Among the reasons for the decline was the fact that large energies could be generated using HEPP, but we lacked powerconditioning tools, and did not have sophisticated techniques for delivering the large currents to loads of interest. AFRL, with its Shiva Star facility, maintained a continuing interest in low-voltage long pulse techniques, including HEPP, but for a short time in the early 90's, only Los Alamos maintained an active HEPP effort in the US. There is renewed interest in HEPP, and among the reasons are that HEPP devices are being rediscovered as compact and flexible sources of pulsed power, and that we have developed some of the necessary tools and sophistication that were lacking in the 80's. HEPP technology attained a high degree of maturity in the U. S. with the advent of the Los Alamos Procyon system in the early 90's. During the 80's a great deal of technology for delivering microsecond scale 
current pulses to interesting loads was developed at the AFRL Shiva facility ${ }^{2}$. At Los Alamos, during the same period, we were making good progress in pulse conditioning using $\mathrm{HE}$ driven opening switches. The combined knowledge of these two efforts were merged in the Procyon HEPP system and in 1994 we achieved our long-term goal of producing over $1 \mathrm{MJ}$ radiation from a microsecond scale plasma z-pinch ${ }^{3}$. Today there are efforts in both government laboratories and the private sector involving HEPP, and we will describe these here.

In the following text I have chosen to break the material into three categories, computational, experimental, and university programs. By doing so, I have focused on the type of work being done rather than the goals of each program. Most of the work mentioned in this paper is also represented elsewhere in the conference, and specific program goals can be ascertained from those papers. Within each category, I have treated the programs one at a time. In reviewing the work, I will reference many papers in this conference (ITC) by principle author, but will not attempt to compile these in a reference list for reasons of space. The reader can find the author, and hence the paper, in the index.

Computational work

One of the fastest growing areas for HEPP is in component modeling and associated tools. Modeling efforts cover the spectrum from empirical models for circuit codes to sophisticated 1-D and 2-D MHD calculations. Represented in these efforts are both government laboratories and the private sector, typically under government sponsorship. Several modeling efforts are currently supporting the AFRL Phillips Site (PS). Among the private sector endeavors is the modeling work done by CARE'N company using their Cajun code. This work is described by Chase, ITC, and has enjoyed considerable success in modeling helical FCG's for the AFRL/PS. In calculations performed either by AFRL/PS personnel or Numerex Inc., the Mach II 2-D MHD code is also seeing considerable use for modeling HEPP components. Noteworthy in this effort are recent predictions by Mach II that the magnetic force acting on the surface of wires in helical FCG's causes a flattening of the wires that gives rise to sideways expansion. This expansion may lead to insulation failure if wires are too closely packed, and may explain certain failures that seem otherwise beyond explanation. Ruden, ITC, describes further AFRL/PS work in assessing the material state of FCG armatures. Those calculations are focused on loss mechanisms. In addition to these FCG efforts, Mach II is now being used to model explosively formed fuse (EFF) opening switches as part of the AFRL/PS program. Numerex Inc. recently put an explosive burn routine into Mach II to support Los Alamos programs ${ }^{4}$, and this has paved the way for the EFF work. Dennis Keefer presents some of these calculations ITC, and it is interesting to compare them with the experimental work that Tasker, at Los Alamos, is also presenting here.

For other work sponsored by AFRL at Eglin AFB, SAIC has developed the FCGSCA code for modeling FCG's. The code is programmed in Visual Basic, is very graphic, and is set up for performing parametric studies easily. This model has met with some successes in matching results from both MK-IX and Ranchito helical FCG's from Los Alamos, and is the subject of a paper ITC by Cornette.

At Los Alamos, we continue to use the Scat95 code to perform HEPP circuit simulation. The main strength of this code is the coupling of a variety of HEPP component models, ranging from helical to coaxial FCG's, with a circuit code for system simulation. Tabular values for inductance and resistance can be inserted as inductors or resistors, allowing experimental curves to be used as circuit elements. In addition, Scat 95 provides a data analysis package that allows quick 
comparison of experimental data with circuit predictions. Another effort at Los Alamos has been the undertaking of a first principles model of helical generators. This is an analytical model that defines inductance using the magnetic vector potential, and is discussed by Fortgang ITC. Another noteworthy modeling effort is a disk FCG model by Reinovsky at Los Alamos ${ }^{5}$. This model uses Gurney theory, with modifications to account for ring-up effects, to drive plates at a prescribed burn velocity along a 2-D contour. It includes field diffusion into conductors and magnetic pressure, allowing flux pocketing to be predicted. We have used this model, along with our CONFUSE code ${ }^{6}$, as one of our principle analysis tools in our collaborative work with VNIIEF (Lindemuth, ITC), as well as to analyze the results from the few disk FCG tests we have performed. Finally, at Los Alamos, we are using one and two-dimensional MHD codes to model various aspects of our high current Ranchero generators. Recent 1-D calculations, for instance, show that the use of $\mathrm{SF}_{6}$ gas in our Ranchero FCG accounts for a considerable retardation of the armature as it approaches the stator, and that the generator exhibits largely 1-D behavior with that factor included.

\section{Experimental Programs}

Both government laboratories and the private sector are also involved in HEPP experimental programs. Although most work is performed as part of government projects, the work being done by Schlumberger in the oil service industry is an exception. Schlumberger has proposed using small CMF devices (patent \# 5505134) to fire slapper detonators down hole for perforating well casings. They are currently considering this option, along with others, for future development.

The U. S. Army Space and Missile Defense Command is presently sponsoring an experimental technology evaluation program. A paper presented by Altgilbers, ITC, describes this effort, and includes some work performed by Explosive Pulsed Power Industries, Inc., a new company in the private sector becoming involved in experimental HEPP work. U. S. tests in this program are conducted at the Energetic Materials Research and Technology Center located at New Mexico Technological University.

In one of the largest experimental efforts in the U. S., the AFRL/PS is working on helical FCG's and first stage pulse compression. The helical FCG effort is aimed at a system that will deliver $\sim 1 \mathrm{~W}$ at $\sim 2 \mathrm{MA}$, and is the subject of a paper by LEHR, ITC, that presents experimental results. The AFRL/PS is also sponsoring explosive-driven opening switch work at Los Alamos, with the goal of performing pulse conditioning on the waveform produced by their helical generator. Los Alamos has developed the sophisticated detonation systems required for this work, and has experience with large high-energy systems. The major challenges for this effort are reducing the size and managing larger voltages in smaller packages. This work is meeting with some success, and is the subject of papers ITC by Kiuttu, Keefer, Degnan, and Tasker.

At Los Alamos, we continue to pursue a wide variety of HEPP endeavors in addition to the work for AFRL/PS just noted. Our thrusts, at this time, are in three principle directions. The first is the continued development of HEPP components. We are currently working on Ranchero FCG's as a 30-80 MA current source to drive hydrodynamic liner loads. Goforth and Tasker, ITC report this work. In addition, we have dedicated a few experiments to the development of the Ranchito generator. This is a mid-sized helical FCG designed to allow high-energy generators, such as Ranchero, to be operated in remote locations without a large capacitor bank to provide the several MA initial current required for these applications. Our second thrust is to continue to apply HEPP techniques to a 
variety of systems. We generally expect the development of a system to be more difficult that any individual component. We have previously mentioned the Procyon system as an example of a very mature system, and we are conducting experiments that couple Ranchero generators to imploding liner loads with fuse opening switches (Goforth, ITC). Another system aspect we are addressing at Los Alamos is using transformers to provide impedance matching for high impedance loads (Fortgang, ITC). In our third thrust, we maintain an active effort in the generation of high magnetic fields for their own sake. ITC we have reports by Brooks and Clark on recent Dirac series experiments in which we used either Los Alamos 1-2 MG systems or VNIIEF MC-1 10 MG systems to perform high field physics experiments. Another series of Dirac experiments is being planned, and experiments and magnetic field requirements currently being defined.

\section{University Programs}

Because of the renewed interest in HEPP, there is some work now being sponsored at universities. All of these efforts are just beginning, and we are looking forward to the availability of information they will provide. For instance, helical FCG modeling is being performed for the Air Force Office of Scientific Research by the Aeronautics and Astronautics Department at the University of Washington ${ }^{5}$.

We have also instituted a considerable effort in the form of a Multidisciplinary University Research Initiative (MURI) on "Explosive-Driven Pulsed Power Generation". This is a joint effort between Texas Tech University and The University of Missouri at Rolla, and one project is described by Kristiansen, ITC. The major research objectives are gaining improved understanding of FCG issues, and developing methods for efficient conversion of FCG outputs to forms suitable for directed energy systems.

Finally, Freeman, ITC, describes a proposed facility for performing HEPP experiments at Texas A\&M University in this conference. Conclusion

At this time, a relatively large fraction of our total effort is being applied to modeling helical FCG's. Although these are among the oldest types of FCG's in use, they are among the most difficult to model. In the meantime, codes like Cajun, FCGSCA, and Scat95 provide convenient PC based platforms for system or component modeling. 1-D and 2-D calculations are performed at Los Alamos using resident MHD codes, and Mach II is being used by a variety of institutions for 2-D MHD component modeling. This is now being applied to EFF opening switches also. Experimentally, there are significant efforts to develop helical FCG's at Los Alamos and AFRL. Los Alamos continues to work on high energy FCG's, with most work going into Ranchero systems at this time. In spite of the relative importance of pulse conditioning, the only active efforts are in developing pulse transformers at Los Alamos, and EFF's, at Los Alamos for both Los Alamos and AFRL programs. Finally, high magnetic field work continues to be performed at Los Alamos.

Although capacitive pulsed power (PP) machines have met with a great deal of success in the U. S., there is still a choice that can be made on a case by case basis between fixed PP machines or HEPP machines. There is usually some number of similar pulses at which the cost of a fixed facility is amortized sufficiently to reduce the cost per test below that of the same number of tests using HEPP. However, a high energy density experiment destroys a significant volume of the apparatus on each test, and the incremental cost increases considerably as the experimental energy range increases. A high experiment rate (number of tests per 
time) may or may not show a break point between techniques, depending on complexity of and survivability of diagnostics. Remote experimental locations and the need for compact systems favor HEPP systems. As renewed interest gives rise to new techniques and goals, the HEPP field should continue to grow.

\section{References}

${ }^{1}$ C.M. Fowler, R.S. Caird, W.B. Garn, An Introduction to Explosive Magnetic Flux Compression Generators, Los Alamos internal report LA-5890-MS, February 1975.

${ }^{2}$ W.L. Baker, et al., Plasma Flow Switch Driven Implosions, Megagauss Fields and Pulsed Power Systems, (Proceedings of the Megagauss Fifth Conference) Nova Science Publishers, Inc, 1990, p.615, Titov and Shvetsov eds.

${ }^{3}$ Goforth et al., Procyon: $18 \mathrm{MJ}, 2-\mu \mathrm{s}$ Pulsed Power System, Proceedings of the Tenth IEEE International Pulsed Power Conference, Albuquerque, New Mexico, 1995, p. 478, Baker and Cooperstein eds.

${ }^{4} \mathrm{M}$. Frese, Numerex Inc., private communication.

${ }^{5}$ A.M. Buyko et al., Results of Russian/US High Performance DEMG Experiment, Proceedings of the Tenth IEEE International Pulsed Power Conference, Albuquerque, New Mexico, 1995, p.269, Baker and Cooperstein, eds. ${ }^{6}$ I.R. Lindemuth R.E. Reinovsky and J.H. Goforth, "Exploding Metallic Foil Fuse Modeling at Los Alamos", (Proceedings of the Megagauss Fifth Conference) Nova Science Publishers, Inc., 1990, p.269, Titov and Shvetsov eds.

${ }^{7}$ B. Udrea and U. Shumlak, Numerical Study of a Magnetic Cumulative Generator, Conference Record - Abstracts, of the 1998 IEEE International Conference of Plasma Science, Juen 1998, Raleigh, North Carolina. 